\title{
Time- and Spatial-Harmonic Content in Electrical Machines and its Application in Fourier-Based Models
}

\author{
Bert Hannon, Peter Sergeant, Member, IEEE, Luc Dupré, Member, IEEE
}

\begin{abstract}
An increasing interest in both efficient electrical machines and more extensive control strategies demands evermore faster simulation tools. One of the modeling techniques that meets this demand is Fourier-based modeling. However, even Fourier-based models may encounter problems related to CPU usage. To cope with these problems the authors present three simple techniques to reduce the computational time of Fourier-based models for synchronous machines. The techniques are mainly based on a qualitative knowledge of which timeand spatial-harmonic orders are present in the machine's magnetic field. The proposed techniques are validated and a benchmark test has been performed. The possible reduction in computational time shows to be very large, for the presented benchmark case study up to a factor 5000 .
\end{abstract}

Index Terms-Electric machines, analytical models, harmonic analysis

\section{INTRODUCTION}

Although the importance of traditional electrical machines, such as grid-connected induction machines, is still very large, the demand for higher efficiencies is pushing electrical engineers towards new machine topologies and more complicated control strategies. The development and implementation of such machines requires a good understanding of their physics and operation. Moreover, the design process of new machines requires a fast and accurate modeling technique, suited for optimization purposes. One modeling technique that has recently gained a lot of attention is Fourierbased modeling [1]-[6]. The reason for its popularity is that Fourier-based modeling is extremely suited for both the study and the design of electrical machines. Indeed, despite their analytical character, which enables low computational times, Fourier-based models are capable of accurately taking into account complex physical phenomena such as eddycurrent reaction field [4], [5] and the slotting effect [5], [6]. Moreover, Fourier-based models also give a good insight in the machine's physics.

B. Hannon is with the Electrical Energy Laboratory, Ghent University, Ghent, Belgium (e-mail: Bert.Hannon@UGent.be)

P. Hannon Sergeant is with the Electrical Energy Laboratory, Ghent University, Ghent, Belgium (e-mail: Peter.Sergeant@UGent.be)

L. Dupré is with the Electrical Energy Laboratory, Ghent University, Ghent, Belgium (e-mail: Luc.Dupre@UGent.be)
As already mentioned, one of the major advantages of Fourier-based models is that they can be very fast. However, if a demand for high accuracy is combined with complex machine topologies, the calculation time of traditional Fourier-based models may become unacceptably high. This is especially true when the model is used for optimization procedures.

With this work the authors want to provide simple techniques that allow decreasing the computational time of Fourier-based models without affecting their accuracy. Because of their relative importance in the segment of innovative electrical machines, the focus in this work is on Permanent-Magnet Synchronous Machines (PMSMs).

The study is mainly based on a preliminary knowledge of which time- and spatial-harmonic combinations will be present in the calculated magnetic field. The results of the conducted study are validated using a Finite-Element Model (FEM). A benchmarking test is per formed to compare the computational time of the standard Fourier-based model with the optimized model.

Before discussing the work, some general assumptions have to be made. As already mentioned, this paper focuses on PMSMs. However, such machines come in a large variety. Although the work applies to a great majority of Fourierbased models for synchronous machines, some restrictions have to be adopted.

A first assumption is that the end effects are neglected. This assumption is common for analytical models and allow for a 2D approximation.

Secondly, the applied current system is assumed to be a balanced system with an odd number of phases.

Fourthly, as in most Fourier-based models, saturation is not taken into account.

Finally, the rotor is assumed to be rotating at synchronous speed. This implies steady-state operation.

The above assumptions are listed as:

- No end-effects

- Balanced system with an odd number of phases

- Winding distribution according to SOS

- No saturation

- Synchronous operation 


\section{FOURIER-BASED ANALYTICAL MODELS}

The goal of this section is to introduce Fourier-based modeling. The discussion starts from the Fourier representation of the magnetic field, as presented in [5]. Obviously, the magnetic field is not only dependent on space but on time as well. When building a Fourier-based model this time dependency can be accounted for directly or through timestepping, both techniques are briefly discussed at the end of this section.

\section{A. Fourier representation of the magnetic field}

Fig. 1 shows a PMSM with a Shielding Cylinder (SC). The latter is a conductive sleeve that is wrapped around the magnets [4], [5]. It is often used to reduce the rotor losses at high-speed operation, but it can also be used to retain the magnets. Although the presented work applies to a broader range of PMSMs, as explained in Section I, the machine in Fig. 1 will be used as an example throughout the entire work.

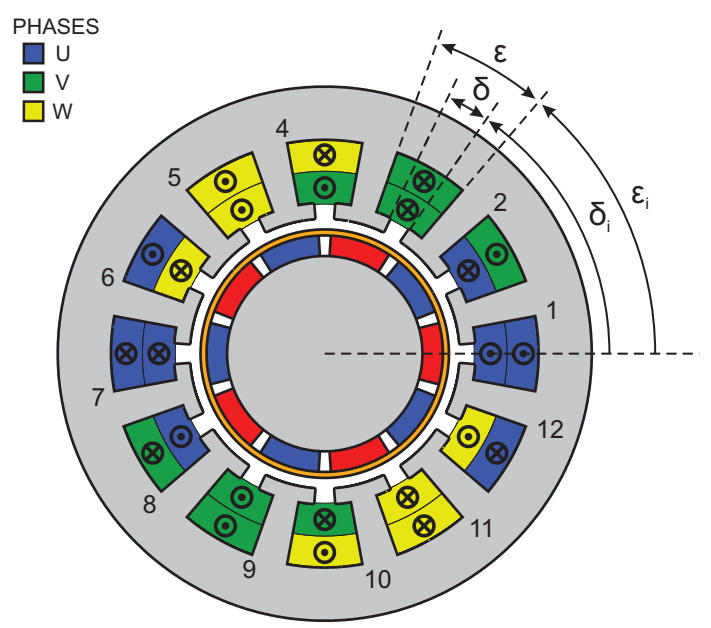

Fig. 1: Geometry of a machine with 12 slots and 5 pole pairs

The magnetic field in an electrical machine has both a time $(t)$ and a spatial dependency. It can easily be seen from Fig. 1 that the shape of the studied machines lends itself to using a cylindrical coordinate system $(r, \phi, z)$ to describe the magnetic field. In the following, the spatial coordinate system is fixed to the stator; $(r, \phi, z)$ is thus a stator reference system. Note that neglection of the end effects means that the magnetic field is independent from the $z$-coordinate.

If the machine is operated in steady-state, the magnetic field has a periodicity over time. This period is called the basic time period $\left(T_{m}\right)$. It is the time the rotor needs to perform one mechanical revolution. It can easily be seen from the machine's geometry that the magnetic field has a spatial periodicity of one revolution, i.e. $2 \pi$ radians in the $\phi$ direction. This period is called the basic spatial period. Obviously, the spatial period can be smaller than $2 \pi$ mechanical radians, but for now that is of little importance.

The magnetic field is mostly expressed using auxiliary quantities such as the magnetic scalar potential $(\psi)$ [1], [2] or the magnetic vector potential (A) [3]-[6]. As already mentioned, the magnetic field, and thereby $\psi$ and $\mathbf{A}$, are independent from $z$ if the end effects are neglected. However, neglecting the end effects also implies that $\mathbf{A}$ only has a $z$ component:

$$
\mathbf{A}=A_{z} \cdot \mathbf{e}_{\mathbf{z}}=A \cdot \mathbf{e}_{\mathbf{z}}
$$

Which of both auxiliary quantities is considered has no effect on the following discussions. In this work the magnetic vector potential was chosen, but a completely analogue reasoning can be made for the scalar potential.

Using the previously mentioned time and spatial periodicities, the magnetic field can be expressed with a Fourier series over time and space:

$$
A(r, \phi, t)=\sum_{n=-\infty}^{\infty} \sum_{k=-\infty}^{\infty} A_{n, k}(r) e^{j\left(k \phi-n \omega_{m} t\right)}
$$

In (2), $n$ is the time-harmonic order and $k$ is the spatialharmonic order. The machine's mechanical rotational speed is denoted as $\omega_{m}$.

$$
\omega_{m}=\frac{2 \pi}{T_{m}}
$$

Because of the fact that the magnetic field has both a time and a spatial dependency, every Fourier coefficient $\left(A_{n, k}(r)\right)$ depends on both the time-harmonic order $n$ and the spatialharmonic order $k$. This means that the time- and spatialharmonic orders should not be regarded separately but as a combination. Such a time- and spatial-harmonic combination is referred to as $(n, k)$. The part of the field that is related to harmonic combination $(n, k)$ is referred to as the $(n, k)$ component of the magnetic field. If $k \phi-n \omega_{m} t$ is assumed constant, the rotational speed of this component can be calculated as:

$$
\frac{\mathrm{d} \phi}{\mathrm{dt}}=\frac{n}{k} \omega_{m}
$$

The rotational speed thus depends on the $n$ to $k$ ratio and can be both positive or negative, as can be seen from (2) and (4).

\section{B. Fourier-based modeling}

The fact that the magnetic field can be written as a Fourier series has been used by a great number of authors to construct analytical models of PMSMs [1]-[6]. In these Fourier-based models, Maxwell's equations and the constitutive relations are used to formulate a differential equation for the magnetic scalar potential or the magnetic vector potential. For the magnetic vector potential this differential equation is written as:

$$
\nabla^{2} \mathbf{A}-\mu \sigma \frac{\partial \mathbf{A}}{\partial t}=-\mu \mathbf{J}_{\text {ext }}-\nabla \times \mathbf{B}_{\text {rem }}
$$


To solve (5) the subdomain technique is applied. This technique divides the machine in a number of regions, called subdomains. The governing equation is greatly simplified in each of the subdomains. Fig. 2 shows the subdomains in a slotted and in a slotless machine. They are indicated with an index $\nu$, ranging from 1 till 4 in the slotless machine and 1 till 5 in the slotted machine. Note that, in slotted machines, every slot and every slot opening is a separate subdomain. These domains are represented by indices $4 i$ and $5 i$, where $i$ refers to the slot number. Subdomain 2 is the SC. Not every machine is equipped with a shielding cylinder, however whether or not a $\mathrm{SC}$ is present in the machine has no influence in this work.

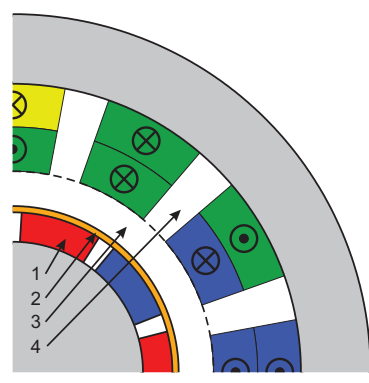

(a) Slotless machine

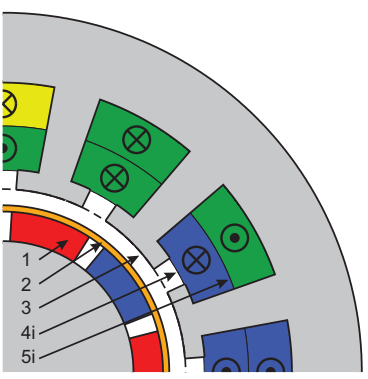

(b) Slotted machine
Fig. 2: Subdomains in slotless and slotted machines

The subdomain technique now consists of two steps. Firstly the simplified differential equations are solved in every subdomain. The resulting solutions for the magnetic vector potential are then indicated by $\mathbf{A}^{(\boldsymbol{\nu})}$. In a second step the integration constants are determined by imposing physical boundary conditions, i.e. Ampère's law and conservation of the magnetic flux. By doing so the different subdomains are linked back together.

The solution of the simplified differential equation is obtained using the separation-of-variables technique. The equation for the magnetic vector potential will thus be written as the multiplication of a $r$-, a $\phi$ - and a $t$-dependent part. The form of these parts depends on the differential equation and the spatial periodicity in the considered subdomain.

The actual form of the $r$-dependent is not of great importance in this work. It is however important to note that the $r$ dependent part contains the integration constants, introduced when solving (5).

In Section II-A, the $\phi$-dependent part of harmonic combination $(n, k)$ was written as $e^{j k \phi}$, based on the assumption that the magnetic field has a basic spatial periodicity of $2 \pi$ mechanical radians. This notation holds in the subdomains that span $2 \pi$ mechanical radians. However, in slotted machines the subdomain technique introduces domains that do not span the entire $2 \pi$ mechanical radians, i.e. the slot openings and the slots. As discussed in [3], [5], [6], the magnetic fields in these subdomains have a basic spatial period of twice their respective opening angles, $2 \delta$ for the slot openings and $2 \varepsilon$ for the slots. The $\phi$-dependent part of the magnetic vector potential is then written as:

$$
\begin{cases}e^{j \frac{u \pi}{\delta}\left(\phi-\delta_{i}\right)} & \text { if } \nu=4 i \\ e^{j \frac{v \pi}{\varepsilon}\left(\phi-\varepsilon_{i}\right)} & \text { if } \nu=5 i\end{cases}
$$

To avoid confusion, a different notation for the spatialharmonic order is used; $u$ and $v$ instead of $k$. Note as well that the starting angles of the slot opening and the slot, shown in Fig. 1, are subtracted from the angular coordinate. This is done to easily comply with the imposed boundary conditions [3], [5], [6].

The spatial division of the machine's geometry has no effect on the basic time period. The $t$-dependent part will always be written as $e^{-j n \omega_{m} t}$.

The above implies that the magnetic vector potential in every subdomain can be written as:

$$
A^{(\nu)}(r, \phi, t)=\sum_{n=-\infty}^{\infty} \sum_{k=-\infty}^{\infty} A_{n, k}^{(\nu)}(r) e^{j\left(\frac{k}{T^{(\nu)}}\left(\phi-\phi_{0}^{(\nu)}\right)-n \omega_{m} t\right)}
$$

Where $T^{(\nu)}$ is the subdomain's spatial period, i.e. $2 \delta$ in the slot openings, $2 \epsilon$ in the slots and $2 \pi$ in the other subdomains. The starting angle of subdomain $\nu$, i.e. $\delta_{i}$ in the slot openings, $\epsilon_{i}$ in the slots and 0 in the other subdomains is indicated as $\phi_{0}^{(\nu)}$.

A more extensive description of the subdomain technique is provided in [1]-[6] and is beyond the scope of this work.

\section{Time harmonics versus time stepping}

In the above, the time-dependency of the magnetic field was directly taken into account, resulting in time-dependent equations for the magnetic vector potential; (7). The main advantage of this approach is that the eddy-current reaction field can be taken into account. Indeed, in order to consider the effect of the eddy-currents in a certain subdomain, the time-derivative term of (5) has to be adopted in its simplified differential equation. This is only possible if the timedependency is directly taken into account. This technique is rather new. It has been used to calculate the field in PMSMs with a shielding cylinder [4], [5] and to compute the eddycurrent losses in the stator conductors [7]. It could also be used to build Fourier-based models of induction machines, which has recently been done by Boughrara et al. [8], [9]. An alternative for the direct time-dependency is taking the time dependency into account through time stepping. The basic time period is then divided in a number of steps. At each of these time instants the current density and the position of the magnets are updated and the magnetic field is computed without taking into account the time-dependency. This method is attractive because it is relatively simple and, as long as the required number of time steps is limited, 
demands little computational time. It has been used by a large number of authors for various machine topologies [1] [3], [6].

Since directly taking into account the time dependency is the most general method, it will be used in the rest of this work. The results apply to models that use time-stepping if the time-harmonic order $(n)$ is assumed to be 1 .

\section{HARMONIC CONTENT IN SYNCHRONOUS ELECTRICAL MACHINES}

In (7), every time- and spatial-harmonic order is considered. However, usually not every harmonic combination is present in the machine's magnetic field. Moreover, the harmonic content of synchronous electrical machines can be predicted. An extensive discussion on this prediction is beyond the scope of this work, but a brief introduction on the topic will be presented in the following so that the results can be used in Section IV.

There are four aspects that define the machine's harmonic content; the permanent magnets, the stator currents, the winding distribution and the machine's geometry. These four aspects can be divided in two categories: the source terms, i.e. the magnets and the stator currents, and the spatial aspects, i.e. the machine's geometry and the winding distribution. The source terms determine which time-harmonic orders are present in the machine's magnetic field. Indeed, they can only introduce time-harmonic orders that are present in their own harmonic spectrum; $h_{m}$ for the magnets and $h_{c}$ for the stator currents. Based on the time harmonic content, the spatial aspects determine the machine's spatial harmonic content. The latter is argued by noting that the machine's magnetic field will be equal but rotated over a certain angle after a given time span. This rotational angle and time span are determined by the machine's spatial aspects.

\section{A. No-load situation}

The above can easily be explained for the no-load case of a machine with identical slots. If $N_{s}$ is the number of slots, the rotor will have rotated over one slot pitch after $\frac{T_{m}}{N_{s}}$ seconds. The magnets are then rotated over $\frac{2 \pi}{N_{s}}$ mechanical radians while the flux produced by these magnets experiences the same magnetic reluctance. This means that, from the stator point of view, the magnetic field will be identical but rotated over $\frac{2 \pi}{N_{s}}$ radians. Note that not every electrical machine is equipped with $N_{s}$ equal slots. However, if the slots are unequal there is always a repetition in the shape of the slots. If there are $N_{s, e q}$ similar sets of $N_{t}$ subsequent slots, the rotational angle becomes $\frac{2 \pi}{N_{s, e q}}$ radians and the time period becomes $\frac{T_{m}}{N_{s, e q}}$ seconds. This can be expressed mathematically:

$$
A\left(r, \phi, t_{0}\right)=A\left(r, \phi+\frac{2 \pi}{N_{s, e q}}, t_{0}+\frac{T_{m}}{N_{s, e q}}\right)
$$

Moreover, the above is valid for every harmonic combination separately:

$$
\begin{aligned}
& A_{n, k}(r) e^{j\left(k \phi-n \omega_{m} t_{0}\right)}= \\
& \quad A_{n, k}(r) e^{j\left(k\left(\phi+\frac{2 \pi}{N_{s, e q}}\right)-n \omega_{m}\left(t_{0}+\frac{T_{m}}{N_{s}, e q}\right)\right)}
\end{aligned}
$$

Considering that $\omega_{m} T_{m}=2 \pi$, (9) implies a relation between the time- and spatial-harmonic orders.

$$
k-n=c N_{s, e q}
$$

where $c$ is an integer.

This means that, for the no-load situation, the time-harmonic orders are determined by the harmonic spectrum of the magnets while the machine geometry, i.e. the slots, determine the present spatial-harmonic orders. The harmonic combinations then have to satisfy (10) while $n \in h_{m}$.

\section{B. Armature-reaction situation}

For the armature-reaction field a similar approach can be made. The time-harmonic orders in the magnetic field are now determined by the harmonic spectrum of the applied currents $\left(n \in h_{c}\right)$. Let $m$ be the number of phases and $\tau$ the machine's period, i.e. the greatest common divisor of the number of pole pairs $(p)$ and the number of slots $\left(N_{s}\right)$. When the machine's winding is determined using the SOS, it contains $\varsigma m \tau$ slot groups (also known as phase belts). Where $\varsigma=1$ or $\varsigma=2$, depending on whether $\frac{N_{s}}{\tau}$ is odd or even. The spatial shift of a slot group corresponds to the time shift of the currents flowing through that slot group. This means that the machine's magnetic field will be equal but rotated over one slot group after $\frac{T_{m}}{m \tau}$ or $\frac{T_{m}}{2 m \tau}$ seconds. That periodicity of the armature-reaction field is mathematically expressed as:

$$
\begin{aligned}
& A_{n, k}(r) e^{j\left(k \phi-n \omega_{m} t_{0}\right)}= \\
& \quad A_{n, k}(r) e^{j\left(k\left(\phi+\frac{2 \pi}{\varsigma m \tau}\right)-n \omega_{m}\left(t_{0}+\frac{T_{m}}{\varsigma m \tau}\right)\right)}
\end{aligned}
$$

From which the following relation between the time- and spatial-harmonic orders is obtained:

$$
k-n=c s m \tau
$$

This means that under armature-reaction conditions, the harmonic combinations in the magnetic field have to satisfy (12) while $n \in h_{c}$.

\section{Load situation}

Finally, since saturation is neglected, the load field is the superposition of the no-load and armature-reaction fields. The time-harmonic orders are now introduced by both the rotor magnets and the stator currents. As mentioned, the effect of the geometry is already incorporated in the winding distribution. This means that, depending on whether $\frac{N_{s}}{\tau}$ is odd or even, the magnetic field is identical but rotated over 
$\frac{2 \pi}{m \tau}$ or $\frac{\pi}{m \tau}$ mechanical radians after $\frac{T_{m}}{m \tau}$ or $\frac{T_{m}}{2 m \tau}$ seconds. Furthermore, under load conditions the harmonic combinations in the magnetic field have to satisfy (13).

$$
\left\{\begin{array}{l}
n \in h_{m} \text { or } n \in h_{c} \\
k-n=c \varsigma m \tau
\end{array}\right.
$$

As a conclusion it can be stated that the harmonic combinations, present in the magnetic field of a synchronous machine under load conditions, can be predicted using (13).

\section{HARMONICS AND FOURIER-BASED MODELS}

A demand for low computational time is one of the major reasons to develop a Fourier-based model. Such models can indeed be very fast. However, their computational time is mainly determined by the amount of integration constants that have to be calculated. Which in turn depends on the amount of harmonic combinations that are taken into account. Since more accuracy implies more harmonic combinations, a strict demand for accuracy may result in unacceptable computational times. This is especially true for models that directly take into account the time dependency and for models which' purpose is an optimization procedure.

The geometry also has an impact on the computational time. Indeed, in slotted machines, every slot opening and every slot is a separate subdomain. This implies that the number of slots greatly affects the amount of integration constants, and thereby the computational time.

The goal of this section is to reduce the computational time of Fourier-based models, without affecting the accuracy. To do so the findings from the previous sections are applied. In a first part the harmonic combinations are reduced using (10), (12) and (13). In the second part the interdependency of the slots is discussed. Thirdly the real nature of the magnetic vector potential is discussed.

\section{A. Harmonic combinations}

When simulating a machine with the use of a Fourierbased model, the number of considered time- and spatialharmonic orders is always limited. The cut-off harmonic orders, i.e. highest time and spatial harmonics that are taken into account, have to be carefully chosen. On the one hand a sufficient amount of harmonic orders is required to obtain accurate results. On the other hand the amount of considered harmonic orders should be as low as possible in order to avoid excessive computational times. However, by applying the results of Section III the amount of harmonic combinations can be reduced without affecting the accuracy. Indeed, depending on the situation, the field components related to harmonic combinations that do not satisfy the requirements imposed in (10), (12) or (13) will be zero. These combinations can thus be disregarded.

Note that the imposed relations between the time- and spatialharmonic combinations were found assuming a basic spatial period of $2 \pi$ mechanical radians. They are therefore not valid in the slot openings and the slots of a slotted machine.

\section{B. Interdependence of the slots}

Although (10), (12) and (13) do not apply to the magnetic vector potential in the slots and the slot openings, the time periodicities found in Section III are still valid for the machine's total magnetic field.

Equation (8) implies that under no-load conditions the magnetic field in slot opening $i+N_{t}$ lags the magnetic field in slot opening $i$ by $\frac{T_{m}}{N_{s, e q}}$ seconds. A relation can then be found between the magnetic vector potential in these slot openings:

$$
A^{(4 i)}\left(r, \phi, t_{0}\right)=A^{\left(4 i+N_{t}\right)}\left(r, \phi+\frac{2 \pi}{N_{s, e q}}, t_{0}+\frac{T_{m}}{N_{s, e q}}\right)
$$

Which is again valid for every harmonic combination separately:

$$
\begin{aligned}
& A_{n, u}^{(4 i)}(r) e^{j\left(\frac{u \pi}{\varepsilon}\left(\phi-\varepsilon_{i}\right)-n \omega_{m} t_{0}\right)}= \\
& \quad A_{n, u}^{\left(4 i+N_{t}\right)}(r) e^{j\left(\frac{u \pi}{\varepsilon}\left(\phi+\frac{2 \pi}{N_{s, e q}}-\varepsilon_{i+N_{t}}\right)-n \omega_{m}\left(t_{0}+\frac{T_{m}}{N_{s, e q}}\right)\right)}
\end{aligned}
$$

and since $\varepsilon_{i+N_{t}}=\varepsilon_{i}+\frac{2 \pi}{N_{s, e q}}$ :

$$
A_{n, u}^{(4 i)}(r)=A_{n, u}^{\left(4 i+N_{t}\right)}(r) e^{-j n \frac{2 \pi}{N_{s}, e q}}
$$

A similar relation can be found between the Fourier coefficients of the slots:

$$
A_{n, v}^{(5 i)}(r)=A_{n, v}^{\left(5 i+N_{t}\right)}(r) e^{-j n \frac{2 \pi}{N_{s}, e q}}
$$

For the armature-reaction and load fields, a similar periodicity was found. Analogously as in the above, the following relations can be found:

$$
A_{n, u}^{(4 i)}(r)=A_{n, u}^{\left(4 i+\frac{N_{s}}{\varsigma m \tau}\right)}(r) e^{-j n \frac{2 \pi}{\varsigma m \tau}}
$$

and

$$
A_{n, v}^{(5 i)}(r)=A_{n, v}^{\left(5 i+\frac{N_{s}}{\varsigma m \tau}\right)}(r) e^{-j n \frac{2 \pi}{\varsigma m \tau}}
$$

Equations (16)-(19) show a relation between the Fourier coefficients of different slot openings and slots. This implies that fewer coefficients have to be calculated using the boundary condition equations, which results in a lower computational time. This lower computational time is again achieved without loss of accuracy.

\section{Real functions}

Although (7) contains complex components, the magnetic vector potential and the magnetic scalar potential are real functions. This implies that the Fourier coefficients linked to harmonic combinations $(n, k)$ and $(-n,-k)$ have to be complex conjugate. For the magnetic vector potential this implies:

$$
A_{n, k}^{(\nu)}(r)=\left(A_{-n,-k}^{(\nu)}(r)\right)^{*}
$$


Note that this is valid for every $\nu$.

The above relation implies that only half of the integration constants have to be calculated, which results in a large reduction in the computational time.

\section{VALIDATION AND BENCHMARKING}

To validate the statements made in Section IV, an example machine is simulated using the model described in [5]. During this simulation only the harmonic combinations satisfying (10) and (12) were considered. The Fourier coefficients of the magnetic field in the slots were calculated using (17) and (19). The machine's parameters are listed in Table I. The magnets are radially magnetized and the applied current is sinusoidal.

TABLE I: Parameters of the studied machine

\begin{tabular}{lll}
\hline Symbol & Parameter & Value \\
\hline$r_{1}$ & Rotor yoke radius & $14.5 \mathrm{~mm}$ \\
$r_{2}$ & Permanent magnet radius & $18.0 \mathrm{~mm}$ \\
$r_{3}$ & Shielding cylinder radius & $18.5 \mathrm{~mm}$ \\
$r_{4}$ & Air gap outer radius & $20.5 \mathrm{~mm}$ \\
$r_{5}$ & Slot outer radius & $30.4 \mathrm{~mm}$ \\
$r_{6}$ & Machine outer radius & $38.0 \mathrm{~mm}$ \\
$N_{s}$ & Number of slots & 12 \\
$w$ & Number of windings per slot & 5 \\
$\delta$ & Slot opening angle & $0.6 \frac{2 \pi}{N} \mathrm{rad}$ \\
$B_{r e m}$ & Remanent magnetic induction & $1.2 \mathrm{~T}$ \\
$p$ & Number of pole pairs & 2 \\
$\varphi_{m}$ & Magnet span & $0.8 \frac{\pi}{p} \mathrm{rad}$ \\
$I$ & Externally imposed current (amplitude) & $50 \mathrm{~A}$ \\
$f$ & Electrical frequency & $1000 \mathrm{~Hz}$ \\
$\sigma_{2}$ & Conductivity of the SC & $4.83 \cdot 10^{7} \Omega \mathrm{m}$ \\
\hline
\end{tabular}

Note that the machine under consideration, shown in Fig. 3, has no semi-closed slots.

The highest time- and spatial-harmonic orders are set to 50. The induction in the air gap can be calculated using the definition of the magnetic vector potential. The result is compared to finite-element calculations in Fig. 4. The good agreement between analytical and finite-element calculations confirms the findings from Sections III and IV.

To get an idea of how the performed study can affect the computational time of Fourier-based models, the integration constants of the machine were computed both with the standard model, which takes into account every possible harmonic combination, and with the optimized model, which only considers the present harmonic combinations. The calculations were performed on a PC with 8 GB RAM and a quad-core processor that has a clock rate of $2.83 \mathrm{GHz}$. Note that the calculations were performed on one core. The resulting computational times are shown in Fig. 5 for different accuracies, i.e. different cut-off harmonics. Fig. 5 also shows the computational time of a finite-element model for the same

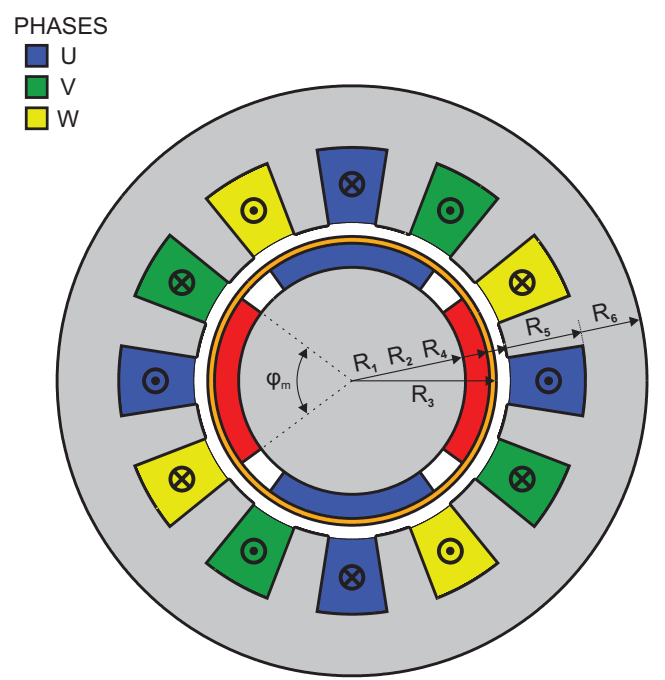

Fig. 3: Geometry of the machine under consideration $\left(N_{s}=\right.$ $12, p=2)$

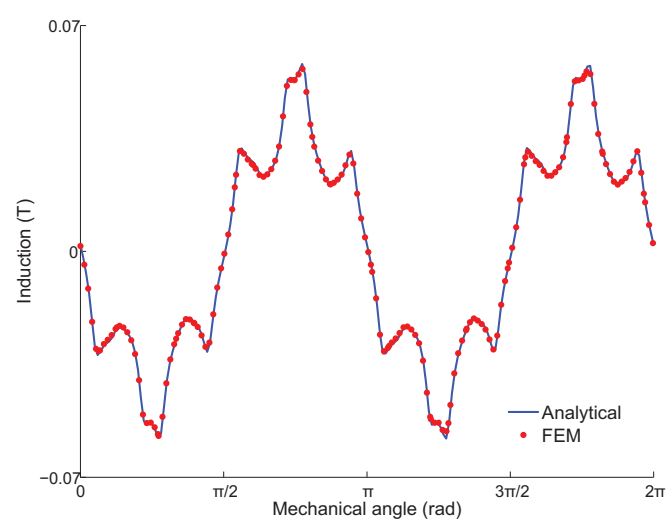

Fig. 4: Radial component of the magnetic induction

machine. This FE model was constructed with a commercial software package and its mesh was optimized to reduce the computational time.

It is clear that the difference between the standard model and the optimized model is very large, the optimized model is up to 5000 times faster. The standard model is even slower than the FEM. Obviously this is unacceptable. Moreover, at the highest accuracies the PC ran out of memory. This explains why there are no computational times for the standard model at very high accuracies. It is thus clear that the results of this work can be used to greatly reduce the computational time of Fourier-based analytical models for synchronous machines.

\section{Vi. CONCLusions}

This work started with an introduction on Fourier-based modeling in Section II and the basics on harmonic content 


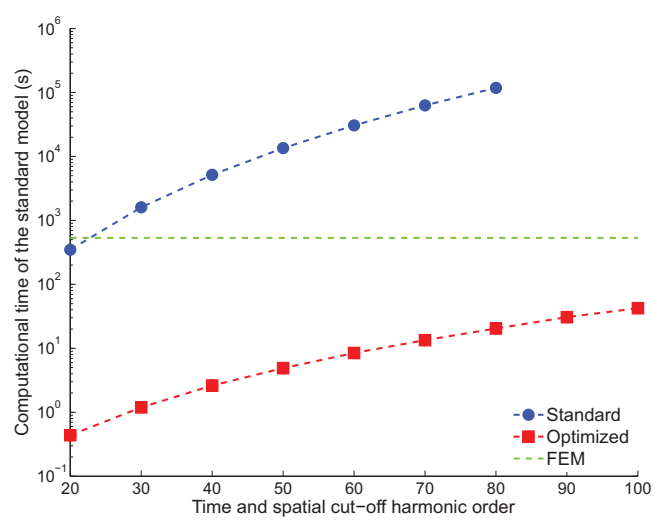

Fig. 5: Comparison of the computational time

of synchronous electrical machines in Section III.

Using the information from these two sections and some general considerations on the magnetic field, three simple techniques to reduce the computational time of Fourierbased models for synchronous machines were proposed in Section IV. A first technique is to only consider the harmonic combinations that are actually present in the magnetic field. Secondly a relation between the magnetic field in different slots can be found. This relation reduces the amount of integration constant that have to be calculated. Finally, it was argued that the auxiliary quantities that describe the magnetic field have to be real. This also imposes a relation between different integration constants.

The above described techniques were successfully validated using a FEM. Finally a benchmark test was done to compare the computational time of the standard Fourier-based model with the optimized model. A huge reduction in computational time was noted. Moreover, it was shown that the memory usage is decreased as well. It should however be noted that the comparison was done for models that directly take into account the time-dependency. The gain will be smaller in models that use time-stepping. Nevertheless, in optimization procedures for example, such a small reduction of the required computational resources might be critical. Moreover, studying the presence of harmonic combinations also results in a better insight in the machine's operation.

The presented work thereby contributes to the development of a faster simulation tool and to the understanding of synchronous machines.

\section{REFERENCES}

[1] Z. Zhu, L. J. Wu, and Z. Xia, "An accurate subdomain model for magnetic field computation in slotted surface-mounted permanent-magnet machines," IEEE Transactions on Magnetics, vol. 46, no. 4, pp. 11001115, Apr. 2010.

[2] Z. Zhu, D. Howe, and C. Chan, "Improved analytical model for predicting the magnetic field distribution in brushless permanent-magnet machines," IEEE Transactions on Magnetics, vol. 38, no. 1, pp. 229238, Jan. 2002.
[3] B. L. J. Gysen, K. Meessen, J. J. H. Paulides, and E. Lomonova, "General formulation of the electromagnetic field distribution in machines and devices using fourier analysis," IEEE Transactions on Magnetics, vol. 46, no. 1, pp. 39-52, Jan. 2010.

[4] S. R. Holm, H. Polinder, and J. Ferreira, "Analytical modeling of a permanent-magnet synchronous machine in a flywheel," IEEE Transactions on Magnetics, vol. 43, no. 5, pp. 1955-1967, May 2007.

[5] B. Hannon, P. Sergeant, and L. Dupré, "2d analytical subdomain model of a slotted PMSM with shielding cylinder," IEEE Transactions on Magnetics, vol. 50, no. 7, pp. 1-11, 2014.

[6] T. Lubin, S. Mezani, and A. Rezzoug, "2-d exact analytical model for surface-mounted permanent-magnet motors with semi-closed slots," IEEE Transactions on Magnetics, vol. 47, no. 2, pp. 479-492, Feb. 2011.

[7] P. Arumugam, F. Dubas, and C. Gerada, "Estimation of eddy current loss in semi-closed slot vertical conductor permanent magnet synchronous machines considering eddy current reaction effect," IEEE Trans. Magn., vol. 49 , no. 10 , pp. $5326-5335$, Oct. 2013

[8] K. Boughrara, N. Takorabet, R. Ibtiouen, and O. Touhami, "Analytical analysis of cage rotor induction motors in healthy, defective and broken bars conditions," IEEE Trans. Magn., vol. Early Acces, pp. 1-19, Aug. 2014.

[9] K. Boughrara, T. Hamiti, and R. Ibtiouen, "2-d analytical prediction of eddy-currents, circuit model parameters and steady states performances in solid rotor induction motors," IEEE Trans. Magn., vol. Early Acces, pp. 1-17, Jul. 2014.

\section{BIOGRAPHIES}

Bert Hannon received the M.Sc. degree in electrotechnical engineering in 2011 from the University College Ghent, Ghent, Belgium. In 2012, he started a Ph.D. study at the Electrical Energy Laboratory of Ghent University. His research interests lies in the analytical modeling of electric machines, with a focus on high-speed machines.

Peter Sergeant received the M.Sc. degree in electromechanical engineering in 2001, and the Ph.D. degree in engineering sciences in 2006, both from Ghent University, Ghent, Belgium. In 2001, he became a researcher at the Electrical Energy Laboratory of Ghent University. He became a postdoctoral researcher at Ghent University in 2006 (postdoctoral fellow of the Research Foundation - Flanders) and at Ghent University College in 2008. Since 2012, he is associate professor at Ghent University. His current research interests include numerical methods in combination with optimization techniques to design nonlinear electromagnetic systems, in particular, electrical machines for sustainable energy applications.

Luc Dupré received the electrical and mechanical engineering degree and the Ph.D. degree in applied sciences from the Ghent University, Belgium, in 1989 and 1995, respectively. In 1989, he joined the Department of Electrical Energy, Systems and Automation, Ghent University, as a research assistant. In 1996, he was a postdoctoral researcher for the Fund of Scientific Research-Flanders and in 1998 for six months a visiting post-doctoral fellow at Istituto Elettrotecnico Nazionale 'Galileo Ferraris', in Torino (Italy). In 2002, he became an associate professor at the Faculty of Engineering and Architecture, Ghent University and since 2006 he is full professor at that faculty. His research interests mainly include numerical methods for low frequency electromagnetism (in particular for electrical machines), modeling and characterization of soft magnetic materials, micromagnetism, inverse problems and optimization in electromagnetism. 